REVISTA DE LA

UNIÓN MATEMÁTICA ARGENTINA

Vol. 59, No. 1, 2018, Pages 143-156

Published online: September 6, 2017

\title{
AN APPLICATION OF PAPPUS' INVOLUTION THEOREM TO CAYLEY-KLEIN PROJECTIVE MODELS
}

\author{
RUBÉN VIGARA
}

\begin{abstract}
Pappus' Involution Theorem is useful for proving incidence relations in the hyperbolic and elliptic planes. This fact is exemplified with the proof of a theorem about a family of 4-gons in the hyperbolic and elliptic planes. This non-Euclidean theorem is also re-interpreted in multiple ways, providing some other theorems for different figures in the hyperbolic plane.
\end{abstract}

\section{INTRODUCTION}

Involutions are a quite useful tool in theorem proving. Many geometric problems admit easier or shorter proofs using involutions (non-geometric problems too, of course - see [16] for an astonishing example!). In the projective context, the most ancient result involving involutions is the following theorem, due to Pappus:

Theorem 1.1 (Pappus' Involution Theorem). The three pairs of opposite sides of a complete quadrangle meet any line not through a vertex in three reciprocal pairs of points of a projective involution.

See [5, p. 49] for a proof. This is a partial version of Desargues' Involution Theorem (see [5, p. 81]; see also [7, pp. 292-293]). A complete quadrangle is the figure in the projective plane that is produced by a set of four points (the vertices of the complete quadrangle), no three of which are collinear, when all the lines joining any two of them (the sides of the complete quadrangle, see Fig. 2) are drawn. Two sides of the complete quadrangle are opposite if they don't share a vertex, and in this case they intersect in a diagonal point of the complete quadrangle. Throughout the paper the word "quadrangle" is always used with this projective meaning, and we identify a complete quadrangle with its set of vertices. With the notation of Fig. 2. the six sides of the complete quadrangle $\mathscr{Q}=\{A, B, C, D\}$ intersect the line $r$ in the six points $E, F, G, H, I, J$. Pappus' Involution Theorem implies that

2010 Mathematics Subject Classification. Primary 51M09, Secondary 51M04.

Key words and phrases. Pappus' Involution Theorem, Cayley-Klein models, non-Euclidean geometry, 4-gons, right-angled hexagons, right-angled pentagons.

This research has been supported by the European Social Fund and Diputación General de Aragón (Grant E15 Geometría), and by Spanish Government's Programa Estatal de Fomento de la Investigación Científica y Técnica de Excelencia (Subprograma Estatal de Generación de Conocimiento, research projects MTM2013-45710-C2 and MTM2016-76868-C2-2-P). 


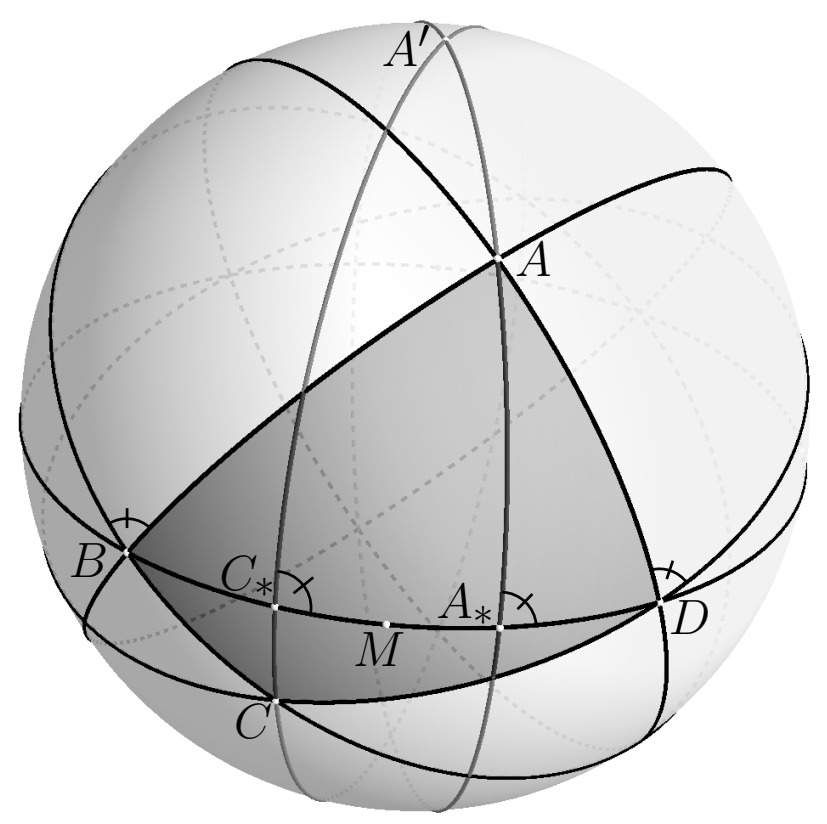

FIGURE 1. Elliptic construction (spherical view).

there is a unique projective involution $\tau_{\mathscr{Q}}: r \rightarrow r$ such that $\tau_{\mathscr{Q}}(E)=F, \tau_{\mathscr{Q}}(G)=H$ and $\tau_{\mathscr{Q}}(I)=J$. The involution $\tau_{\mathscr{Q}}$ is the quadrangular involution induced by $\mathscr{Q}$ in $r$.

During the author's research [15], Pappus' Involution Theorem was found as an extremely powerful tool for proving incidence relations in the hyperbolic and elliptic planes in the framework of Cayley-Klein models, and the first purpose of this article is to exhibit an example of this fact. This is done in Section 3, where a quadrangular involution is used to prove Theorem 1.2 below in a quite simple manner. A 4-sided polygon in the Euclidean, elliptic or hyperbolic plane is called a 4-gon. A 4-gon is diametra 1 if it has two right angles located at opposite vertices.

Theorem 1.2. Let $A B C D$ be a diametral 4-gon in the hyperbolic or elliptic planes with right angles at $B$ and $D$. Let $A_{*}, C_{*}$ be the orthogonal projections of the points $A, C$ into the diagonal $B D$, respectively. A midpoin $t^{2}$ of the segment $\overline{B D}$ is also a midpoint of the segment $\overline{A_{*} C_{*}}$ (see Fig. 1).

\footnotetext{
${ }^{1}$ In the Euclidean case it is a cyclic 4-gon with a diagonal which is a diameter of the circumcycle.

${ }^{2}$ Note that we have written "a midpoint" instead of "the midpoint". Euclidean or hyperbolic segments have undoubtedly a unique midpoint (the midpoint). Since elliptic lines are closed, in the elliptic case this concept is subtler. For convenience, we consider that an elliptic segment with endpoints $A$ and $B$ has two midpoints: the two points collinear with $A$ and $B$ and equidistant from $A$ and $B$.
} 
This theorem also holds in the Euclidean plane and therefore it is a theorem of Bolyai's absolute geometry ${ }^{3}$ In the Euclidean context, it would be a reformulation of [10, problem II.214].

Since projective geometry is a general theory that includes many others, such as Euclidean, affine, hyperbolic and elliptic geometries, for example, it is wellknown that any projective theorem is a general version of many other theorems in all those other settings. In the planar case, in the same way that a projective theorem involving conics can be seen in the Euclidean plane as a set of different theorems for the different conic sections, it can be seen also as a set of theorems for different figures in the hyperbolic and elliptic planes. This idea was used by Thurston [11, Ch. 2] for deducing, at one stroke, the trigonometry of many different figures in the hyperbolic plane: the generalized triangles (see also [4), which are obtained by modifying the relative position of a projective triangle with respect to the absolute conic of the model. Using Thurston's trick, a unique projective proof of a non-Euclidean theorem about triangles provides a bunch of different nonEuclidean theorems, slightly different from the original one, which we call projective variations of the original one. Another purpose of the paper is to illustrate this remarkable property of Cayley-Klein models, that seems to be not very well known, by exploring the projective variations of Theorem 1.2 This is done in Sec. 4 , where five such projective variations (Theorems 4.2 to 4.6) are presented.

Before that, in Sec. 2 the basic tools from projective geometry and Cayley-Klein projective models to be used in the subsequent sections are introduced. We finish in Sec. 5w with some questions that naturally arise from the previous theorems. In particular, we present an Euclidean higher-dimensional generalization of Theorem 1.2 . In all figures right angles are denoted with the symbol $\not x$.

\section{Cayley-Klein models}

It is assumed that the reader is familiar with the basic concepts of real and complex planar projective geometry: the projective plane and its fundamental subsets (points, lines, pencils of lines, conics), and their projectivities. Nevertheless, we give a brief review of some concepts and results needed for a better understanding of Sections 3 and 4 For the rigurous definitions and proofs we refer to [5], [14] or [8], for example. It is also assumed that the reader has some elementary background in non-Euclidean planar geometry (see [6], [11, or the elementary and delightful memoir 9] for example).

Although we work mostly with real elements, the real projective plane $\mathbb{R P}^{2}$ is considered standardly embedded in the complex projective plane $\mathbb{C P}^{2}$.

If $A, B$ are two different points in the projective plane, $A B$ denotes the line joining them. If $a, b$ are two different lines, or a line and a conic, in the projective plane, $a \cdot b$ is their intersection set.

\footnotetext{
3In fact, since Theorem 1.2 only involves the notions of "perpendicularity" and of "midpoint", it makes sense in a much more general framework like Bachmann's theory of metric planes [2], or even in a weaker axiom system than that for metric planes, as that of Hjelmslev groups [3], for example. This was pointed out to the author by a referee.
} 


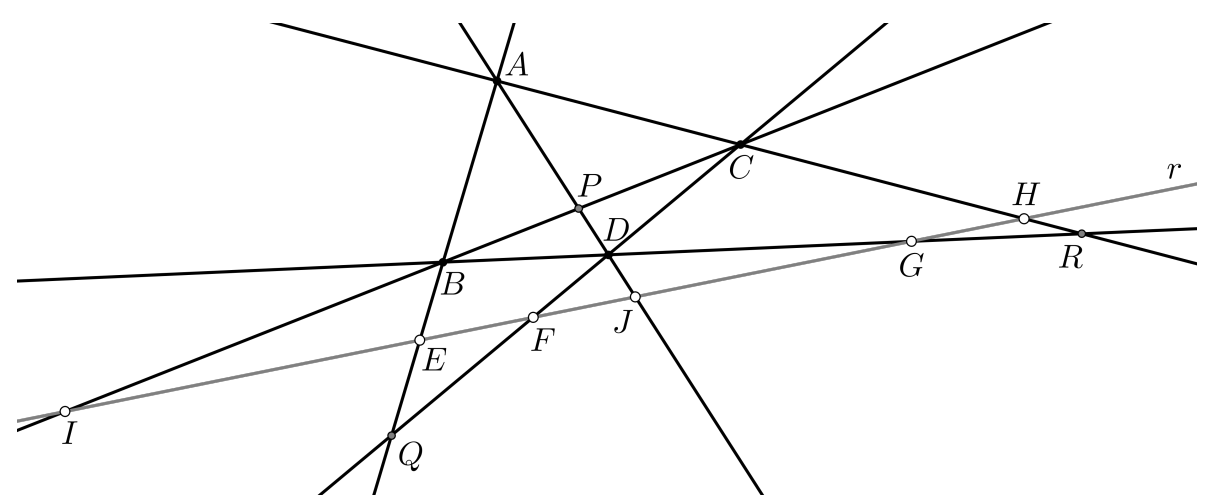

Figure 2. Quadrangles. Pappus' Involution Theorem.

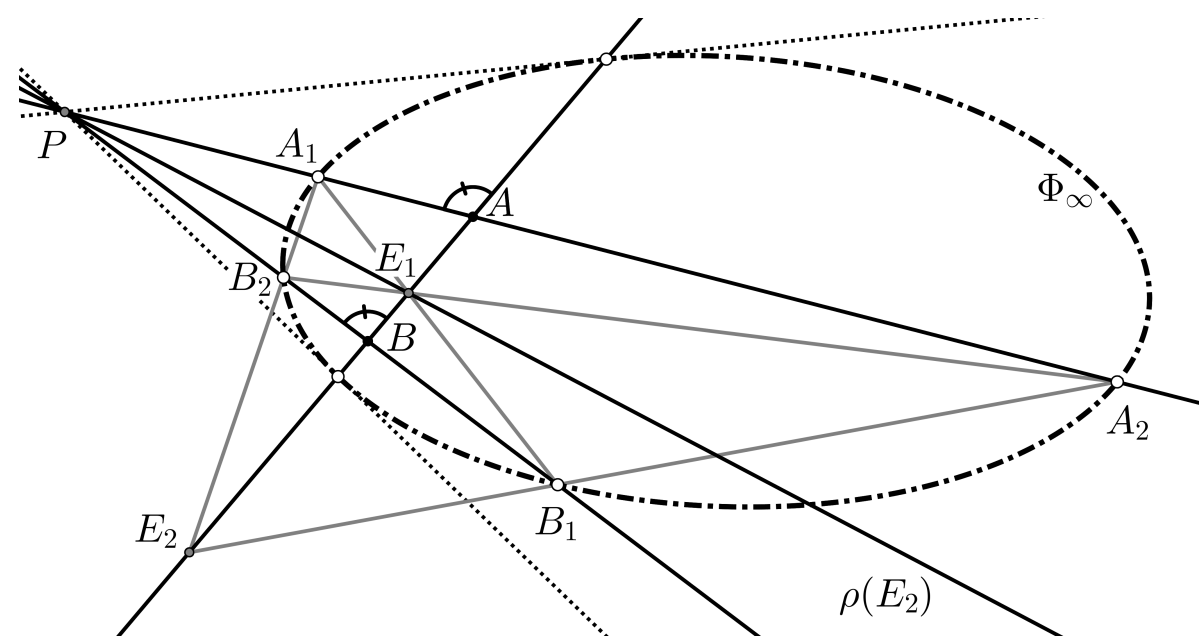

FiguRE 3. Midpoint and orthogonal bisector of a hyperbolic segment.

Let us review the construction of the projective models of the hyperbolic and elliptic planes. We will not give a full construction of these models: we will just show the projective interpretation of the basic geometric concepts that will be needed later. Detailed constructions of the models can be found in [6, 8, 9], for example.

For constructing the non-Euclidean planar models, a non-degenerate conic $\Phi_{\infty}$ is fixed (the absolute conic) such that the polar of each real point with respect to $\Phi_{\infty}$ is a real line. An equivalent formulation of this property is to require, working with homogeneous coordinates in $\mathbb{C P}^{2}$, that $\Phi_{\infty}$ can be expressed by an equation with real coefficients. Such a conic can be of two kinds: a real conic, if it has real points; or an imaginary conic, if it has no real points (see [14, vol. II, p. 186]). 
When $\Phi_{\infty}$ is a real conic, the interior points of $\Phi_{\infty}$ constitute the hyperbolic plane, and when $\Phi_{\infty}$ is an imaginary conic the whole $\mathbb{R} \mathbb{P}^{2}$ constitutes the elliptic plane. We use the common term the non-Euclidean plane either for the hyperbolic plane or for the elliptic plane. Geodesics in these models are given by the intersection with the non-Euclidean plane of real projective lines. If $A, B$ are two different points in the non-Euclidean plane, $\overline{A B}$ denotes a segment having $A$ and $B$ as endpoints. Although in the hyperbolic case points on $\mathbb{R P}^{2}$ not interior to $\Phi_{\infty}$ are not points of the hyperbolic plane, they are of geometric interest for the model (see [11]). For instance, via the absolute polarity (the polarity with respect to $\Phi_{\infty}$ ), each point exterior to $\Phi_{\infty}$ parametrizes a line of the hyperbolic plane (its polar line). Therefore, we will talk about points or lines in a purely projective sense, even if the referred elements are imaginary or, in the hyperbolic case, not interior to $\Phi_{\infty}$.

Let us denote by $\rho$ the absolute polarity. Given a point $P$ and a line $p, \rho(P)$ and $\rho(p)$ are the polar of $P$ and the pole of $p$ with respect to $\Phi_{\infty}$, respectively.

The polarity $\rho$ induces a natural involution on any line $p$ not tangent to $\Phi_{\infty}$ (resp. on any pencil of lines passing through a point $P$ not in $\Phi_{\infty}$ ): the conjugacy involution. This involution sends each point $P \in p$ (resp. each line $p$ through $P$ ) to its conjugate, which is defined as $p \cdot \rho(P)$ (resp. the line $P \rho(p)$ ). The double points of the conjugacy involution on $p$ are the two points on $p \cdot \Phi_{\infty}$. Equivalently, the double lines of the conjugacy involution on the pencil of lines through $P$ are the two lines tangent to $\Phi_{\infty}$ through $P$. Two lines whose intersection point lies in the non-Euclidean plane are perpendicular if they are conjugate with respect to $\Phi_{\infty}$, that is, if each one contains the pole of the other with respect to $\Phi_{\infty}$. If $A$ is a point of the non-Euclidean plane, $a$ is a line through $A$ and $A^{\prime}$ is the conjugate point of $A$ in $a$ with respect to $\Phi_{\infty}$, then $\rho\left(A^{\prime}\right)$ is the line perpendicular to $a$ through $A$ and conversely.

Let $A, B$ be two points on the non-Euclidean plane and take $P=\rho(A B)$. Let $a$ and $b$ be the lines joining $P$ with $A$ and $B$, respectively. Each of the lines $a, b$ has two (perhaps imaginary) different intersection points with $\Phi_{\infty}$. Let $\left\{A_{1}, A_{2}\right\}$ and $\left\{B_{1}, B_{2}\right\}$ be the intersection sets of $a$ and $b$ with $\Phi_{\infty}$ respectively. Since the complete quadrangle $\mathscr{Q}=\left\{A_{1}, A_{2}, B_{1}, B_{2}\right\}$ is inscribed in $\Phi_{\infty}$ the points

$$
E_{1}=A_{1} B_{1} \cdot A_{2} B_{2} \quad \text { and } \quad E_{2}=A_{1} B_{2} \cdot A_{2} B_{1}
$$

lie on $A B$. We say that $E_{1}$ and $E_{2}$ are the midpoints of the segment $\overline{A B}$. Note that this definition of the midpoints of a segment is projective. Moreover, we could talk about midpoints of a projective segment with respect to a conic in a strictly projective context, as it will be done in the statement of Theorem 4.1 This definition can be interpreted in multiple ways (see [6]) in the non-Euclidean context. For example:

\footnotetext{
${ }^{4}$ While the concept of "segment" in the Euclidean or non-Euclidean context is rather intuitive, to define "segment" in a complex projective setting is not a trivial task. For our purposes, it suffices to identify the projective segment $\overline{A B}$ with the set $\{A, B\}$.
} 


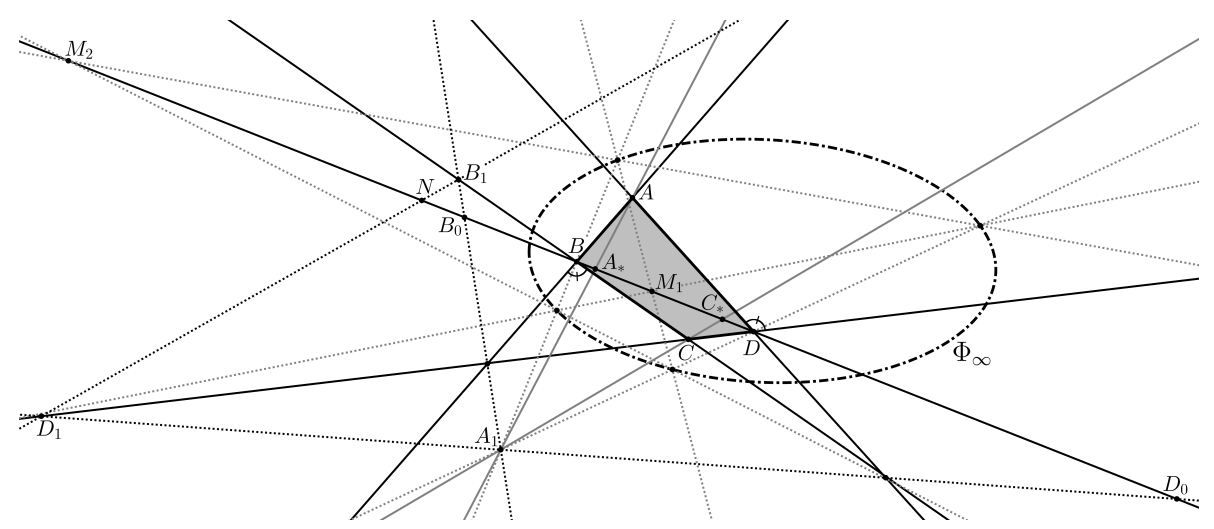

Figure 4. Hyperbolic construction (projective view).

- If $\Phi_{\infty}$ is imaginary, the points $A_{1}, A_{2}, B_{1}, B_{2}$ are also imaginary and $E_{1}$ and $E_{2}$ are the two points of $A B$ which are equidistant from $A$ and $B$ in the elliptic plane.

- If $\Phi_{\infty}$ is a real conic and $A$ and $B$ are interior to $\Phi_{\infty}$, exactly one of the two points $E_{1}, E_{2}$, say $E_{1}$, is interior to $\Phi_{\infty}$ : it is the midpoint of the hyperbolic segment $\overline{A B}$ (Fig. 3 ). The other point $E_{2}$ is the pole of $P E_{1}$, which is the orthogonal bisector of $\overline{A B}$.

- If $\Phi_{\infty}$ is a real conic and $A B$ is exterior to $\Phi_{\infty}$, its pole $P$ is interior to $\Phi_{\infty}$, and the lines $P E_{1}, P E_{2}$ are the two bisectors of the hyperbolic angle $\widehat{a b}$.

By construction, since $E_{1}$ and $E_{2}$ are diagonal points of the complete quadrangle $\mathscr{Q}$, the points $A, B, E_{1}, E_{2}$ form a harmonic set, which in terms of cross-ratios is equivalent to $\left(A B E_{1} E_{2}\right)=-1$. Since $\mathscr{Q}$ is inscribed in $\Phi_{\infty}$, the projective triangle $P E_{1} E_{2}$ is self-polar with respect to $\Phi_{\infty}$, and this implies that $E_{1}$ and $E_{2}$ are conjugate to each other in $A B$. Therefore, if $U$ and $V$ are the two intersection points of $A B$ with $\Phi_{\infty}$, then $\left(U V E_{1} E_{2}\right)=-1$. A useful characterization of midpoints is the following (see [15]):

Lemma 2.1. If $C, D$ are two points of $A B$ such that

$$
(A B C D)=(U V C D)=-1
$$

then $\{C, D\}=\left\{E_{1}, E_{2}\right\}$.

Proof. Let $C, D$ be two points of $A B$ such that $(A B C D)=(U V C D)=-1$. Take the harmonic involutions $\tau_{E_{1} E_{2}}$ and $\tau_{C D}$, of $A B$ with fixed points $E_{1}, E_{2}$ and $C, D$ respectively. The composition $\tau_{E_{1} E_{2}} \circ \tau_{C D}$ fixes the points $U, V, A, B$ and so it must be the identity on $A B$. This implies that $\left\{E_{1}, E_{2}\right\}=\{C, D\}$. 


\section{Proof of Theorem 1.2}

Let $A B C D$ be a 4-gon in the non-Euclidean plane such that the lines $A B$ and $A D$ are conjugate to $B C$ and $D C$ respectively with respect to $\Phi_{\infty}$ (Fig. 4). This means that $B_{1}=\rho(A B)$ and $D_{1}=\rho(A D)$ belong to $B C$ and $D C$, respectively.

Let $a$ be the line $B D$, and take $A_{1}=\rho(a)$. Thus, $A_{*}=a \cdot A A_{1}$ and $C_{*}=a \cdot C A_{1}$.

Let $B_{0}$ and $D_{0}$ be the intersection points with $a$ of the lines $A_{1} B_{1}$ and $A_{1} D_{1}$, respectively. The triangle $A_{1} B_{1} D_{1}$ is the polar triangle of $A B D$. Hence, $B_{0}$ and $D_{0}$ are the conjugate points of $B$ and $D$ in $a$ with respect to $\Phi_{\infty}$, respectively. The point $N=a \cdot B_{1} D_{1}$ is the pole of $A A_{1}$, and so it is the conjugate point of $A_{*}$ in $a$ with respect to $\Phi_{\infty}$.

Let $M_{1}, M_{2}$ be the midpoints of $\overline{B D}$, and consider the complete quadrangle $\mathscr{Q}=\left\{C, A_{1}, B_{1}, D_{1}\right\}$.

Consider the following three involutions in $a$ :

- the conjugacy involution $\rho_{a}$ induced by $\rho$ in $a$;

- the quadrangular involution $\tau$ induced by $\mathscr{Q}$ in $a$; and

- the harmonic involution $\sigma$ in $a$ with respect to $M_{1}$ and $M_{2}$.

The quadrangular involution $\tau$ sends $B, D$ and $C_{*}$ into $D_{0}, B_{0}$ and $N$, respectively. This implies that the composition $\rho_{a} \tau$ sends $B, D, B_{0}$ and $D_{0}$ into $D, B, D_{0}$ and $B_{0}$, respectively. Since

$$
\left(B_{0} D_{0} M_{1} M_{2}\right)=\left(\rho_{a}(B) \rho_{a}(D) \rho_{a}\left(M_{2}\right) \rho_{a}\left(M_{1}\right)\right)=\left(B D M_{2} M_{1}\right)=-1,
$$

then $\sigma\left(B_{0}\right)=D_{0}$. The projectivities $\sigma$ and $\rho_{a} \tau$ of $a$ agree over at least three different points of $a$, and so they coincide. Hence, $\sigma\left(C_{*}\right)=\rho_{a} \tau\left(C_{*}\right)=\rho_{a}(N)=A_{*}$. If the points $A_{*}$ and $C_{*}$ coincide, they coincide with a double point $M_{1}$ or $M_{2}$ of $\sigma$ and the result trivially holds. If $A_{*} \neq C_{*}$, they are harmonic conjugates with respect to $M_{1}$ and $M_{2}$, and therefore

$$
\left(A_{*} C_{*} M_{1} M_{2}\right)=-1 .
$$

If $U$ and $V$ are the intersection points of $a$ with $\Phi_{\infty}$,

$$
\left(U V M_{1} M_{2}\right)=-1
$$

since $M_{1}$ and $M_{2}$ are the midpoints of $\overline{B D}$. By Lemma 2.1 the points $M_{1}$ and $M_{2}$ are the midpoints of $\overline{A_{*} C_{*}}$.

\section{Projective variations of Theorem 1.2}

Disguised in the proof of Theorem 1.2 there is, in fact, a proof of the following projective theorem:

Theorem 4.1. Let $\mathscr{Q}$ be a complete quadrangle in the projective plane with vertices $A, B, C, D$ in general position with respect to $\Phi_{\infty}$ (vertices and diagonal points not in $\Phi_{\infty}$, sides and diagonal line $\$^{5}$ not tangent to $\left.\Phi_{\infty}\right)$ such that the lines $A B$ and $A D$ are conjugate to $B C$ and $D C$ respectively with respect to $\Phi_{\infty}$. Let $A_{1}$ be the pole of $B D$, and let $A_{*}$ and $C_{*}$ be the intersection points of $A A_{1}$ and $C A_{1}$, respectively,

\footnotetext{
${ }^{5}$ The diagonal lines of a complete quadrangle are the lines joining diagonal points.
} 


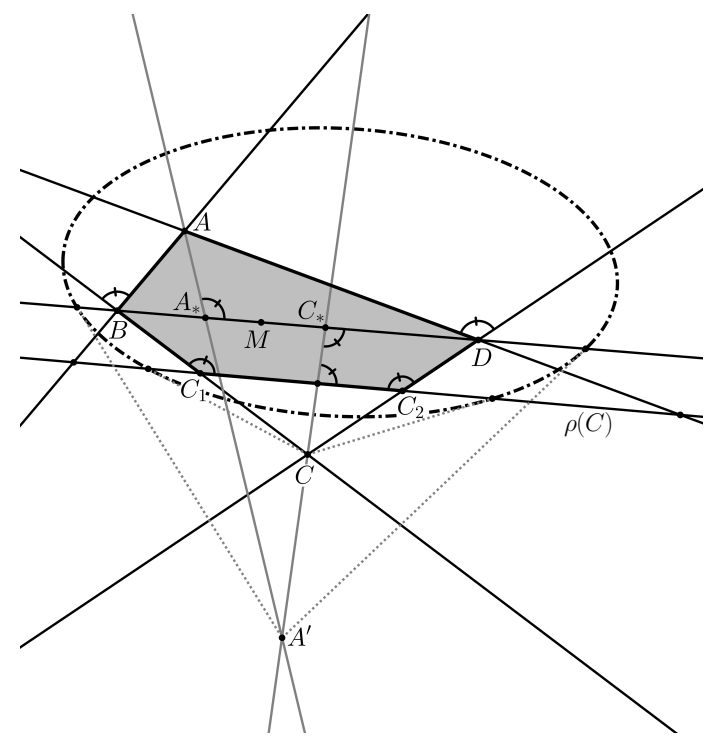

FiguRE 5. 4-right pentagon I.

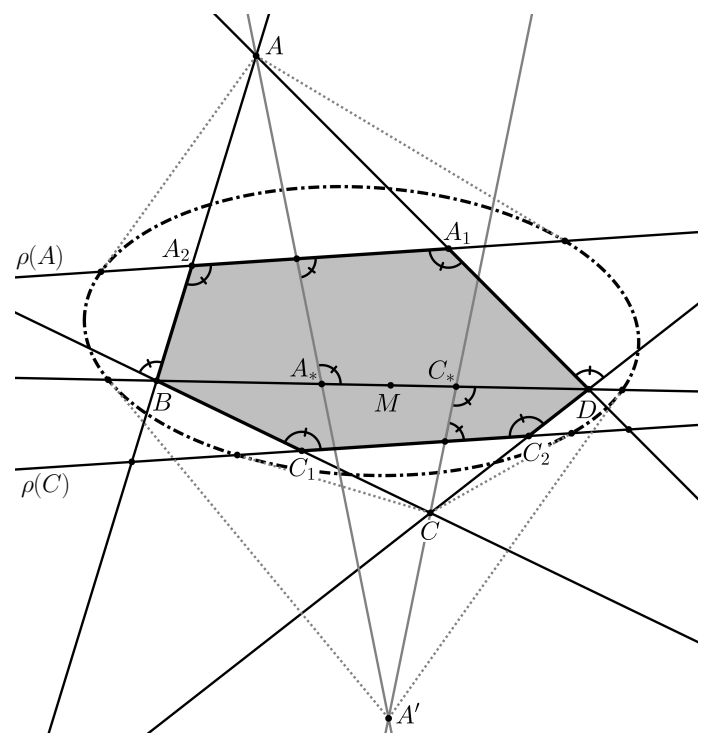

FIGURE 6. Right-angled hexagon I. 


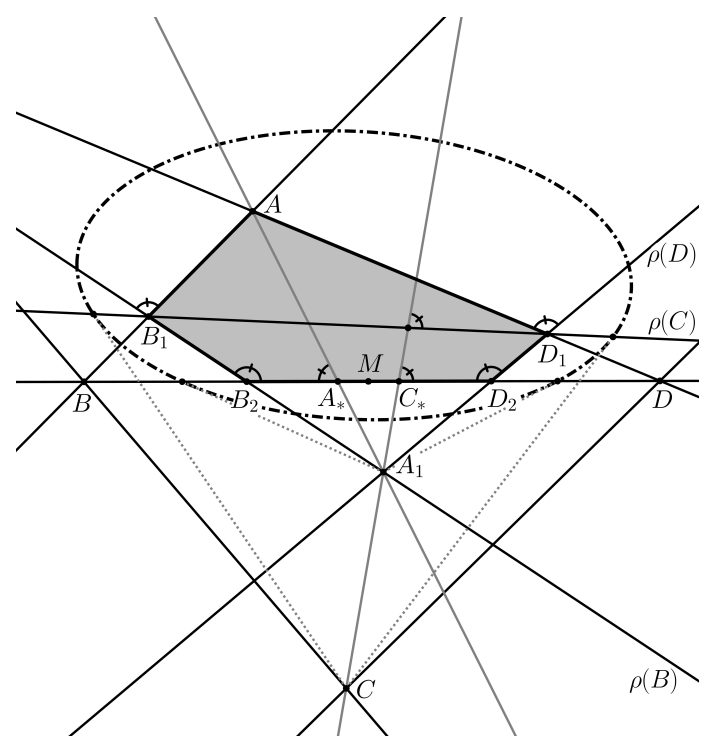

FigURE 7. 4-right pentagon II.

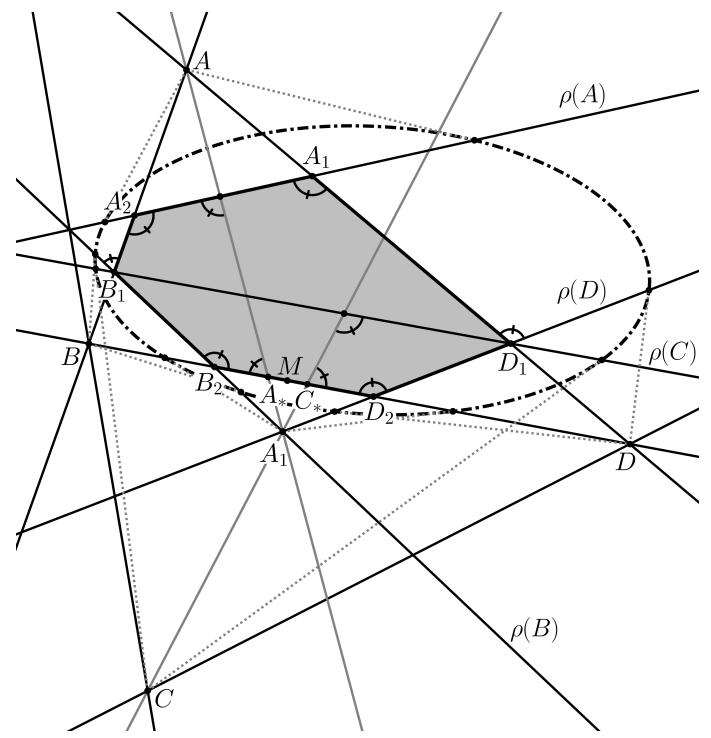

FiguRE 8. Right-angled hexagon II. 
with $B D$. The midpoints of $\overline{A_{*} C_{*}}$ with respect to $\Phi_{\infty}$ (as defined projectively in Section 2) are also the midpoints of $\overline{B D}$ with respect to $\Phi_{\infty}$.

Assume that $A B C D$ is a diametral 4-gon in the hyperbolic plane, as the one depicted in Fig. 4. After changing the relative position of $A, B, C, D$ with respect to $\Phi_{\infty}$ and applying Theorem 4.1 to the resulting figure, some projective variations of Theorem 1.2 are obtained.

4.1. 4-right pentagon. If in the 4-gon $A B C D$ it is assumed that the vertex $C$ lies outside the absolute conic while the rest of vertices remain inside $\Phi_{\infty}$, the polar of $C$ appears in the figure as the common perpendicular to the lines $C B$ and $C D$. The hyperbolic polygon that appears is a 4-right pentagon: a hyperbolic pentagon with, at least, four right angles at the vertices different from $A$ (Fig. 5). In this case, Theorem 4.1 implies:

Theorem 4.2. In the hyperbolic 4-right pentagon $A B C_{1} C_{2} D$, with non-right angle at most at $A$, let $A_{*}$ be the orthogonal projection of $A$ into $B D$, and let $C_{*}$ be the intersection of $B D$ with the common perpendicular to $B D$ and $C_{1} C_{2}$. The midpoint of $\overline{B D}$ is also the midpoint of $\overline{A_{*} C_{*}}$.

4.2. Right-angled hexagon. If in the previous figure the vertex $A$ is pushed out of $\Phi_{\infty}$, while $B$ and $D$ remain interior to $\Phi_{\infty}$, the polar of $A$ becomes part of the figure as the common perpendicular to the lines $A B$ and $A D$. The figure that appears is a right-angled hexagon: an hexagon in the hyperbolic plane with six right angles as the one depicted in Fig. 6. With the notation of this figure, the traslation of Theorem 4.1 for this configuration is:

Theorem 4.3. Let $A_{1} A_{2} B C_{1} C_{2} D$ be a hyperbolic right-angled hexagon. Let $A_{*}$ be the intersection point with $B D$ of the common perpendicular to $B D$ and $A_{1} A_{2}$, and let $C_{*}$ be the intersection point with $B D$ of the common perpendicular to $B D$ and $C_{1} C_{2}$. The midpoint of $\overline{B D}$ is also the midpoint of $\overline{A_{*} C_{*}}$.

4.3. 4-right pentagon II. If, after pushing $C$ out of $\Phi_{\infty}$ for obtaining the 4-right pentagon of Fig. 5 the points $B$ and $D$ are also pushed out of $\Phi_{\infty}$ while the line $B D$ remains secant to $\Phi_{\infty}$ and $A$ remains interior to $\Phi_{\infty}$, the polars of $B$ and $D$ appear in the figure, drawing with the lines $A B, A D$ and $B D$ another 4-right pentagon $A B_{1} B_{2} D_{2} D_{1}$ as the one of Fig. 7. Because the pole of $A B$ is collinear with $B$ and $C$, the polars of $B$ and $C$ intersect at the point $B_{1}$ lying in $A B$, which is also the conjugate point of $C_{1}=B C \cdot \rho(C)$ in $\rho(C)$ with respect to $\Phi_{\infty}$. In the same way, the polars of $C$ and $D$ intersect at the point $D_{1}$ lying in $A D$ which is the conjugate point of $C_{2}=C D \cdot \rho(C)$ in $\rho(C)$ with respect to $\Phi_{\infty}$. On the other hand, the polars of $B$ and $D$ intersect $B D$ at $B_{2}$ and $D_{2}$, respectively, which are the conjugate points of $B, D$ respectively in $B D$ with respect to $\Phi_{\infty}$. The pentagon $A B_{1} B_{2} D_{2} D_{1}$ is a 4 -right pentagon with right angles at all its vertices with the unique possible exception of $A$. By Lemma 2.1 and (3.1), the midpoints of $\overline{B D}$ are also the midpoints of $\overline{B_{2} D_{2}}$. With the notation of Fig. 7

Theorem 4.4. Let $A B_{1} B_{2} D_{2} D_{1}$ be a hyperbolic 4-right pentagon with right angles at the vertices $B_{1}, B_{2}, D_{1}, D_{2}$. Let $A_{*}$ be the orthogonal projection of $A$ into $B_{2} D_{2}$, 


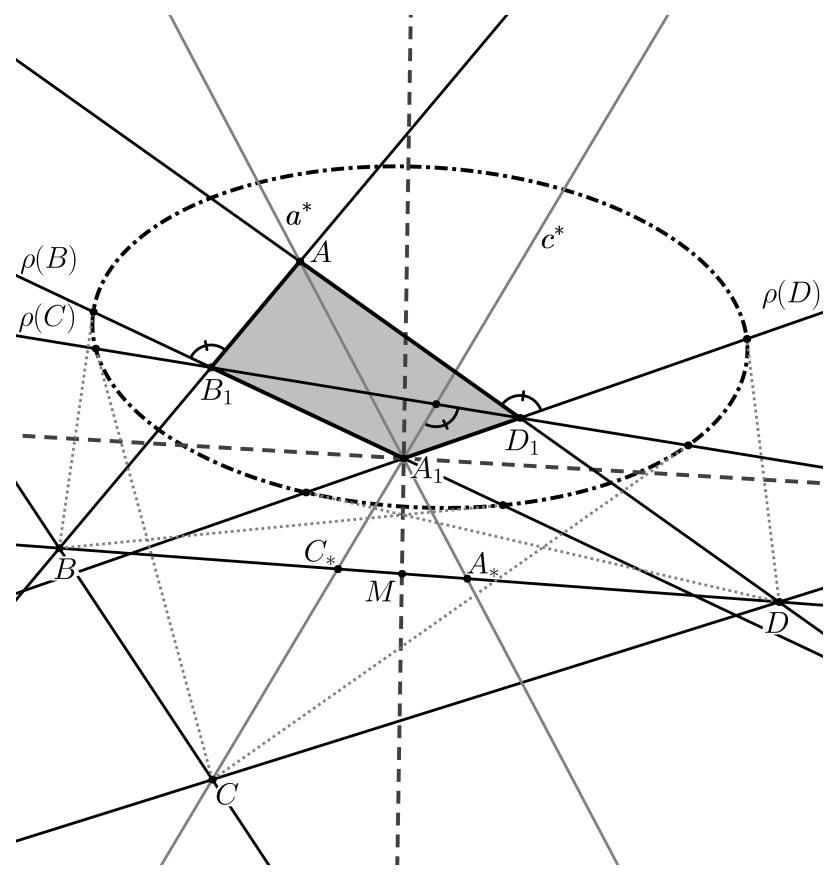

FiguRE 9. Hyperbolic quadrangle revisited.

and let $C_{*}$ be the intersection with $B_{2} D_{2}$ of the common perpendicular to $B_{1} D_{1}$ and $B_{2} D_{2}$. The midpoint of $\overline{B_{2} D_{2}}$ is also the midpoint of $\overline{A_{*} C_{*}}$.

4.4. Right-angled hexagon II. If in the previous figure the point $A$ is also pushed out of the absolute conic, a theorem similar to Theorem 4.4 for right-angled hexagons is obtained. With the notation of Fig. 8

Theorem 4.5. Let $A_{1} A_{2} B_{1} B_{2} D_{2} D_{1}$ be a hyperbolic right-angled hexagon. Let $a_{*}$ and $c_{*}$ be the common perpendiculars to $B_{2} D_{2}$ and $A_{1} A_{2}$ and to $B_{2} D_{2}$ and $B_{1} D_{1}$, respectively, and let $A_{*}$ and $C_{*}$ be the intersection points of $a_{*}$ and $c_{*}$, respectively, with $B_{2} D_{2}$. The midpoint of $\overline{A_{*} C_{*}}$ is the midpoint of $\overline{B_{2} D_{2}}$.

4.5. Quadrangle II. If in the configuration "4 right-pentagon II" (Fig. 7) the points $B, D$ are moved until the line $B D$ is exterior to $\Phi_{\infty}$, the point $A_{1}$ becomes interior to $\Phi_{\infty}$ and $A B_{1} A_{1} D_{1}$ is a hyperbolic diametral 4-gon with right angles at the opposite vertices $B_{1}, D_{1}$. After a reinterpretation of the points $A_{*}, C_{*}, M_{1}, M_{2}$ for this figure, the following theorem is obtained (see Fig. 9):

Theorem 4.6. Let $A B_{1} A_{1} D_{1}$ be a hyperbolic diametral 4-gon with right angles at the opposite vertices $B_{1}, D_{1}$. Consider the line $a_{*}=A A_{1}$ and the line $c_{*}$ perpendicular to $B_{1} D_{1}$ through $A_{1}$. The angle-bisectors of $\widehat{B_{1} A_{1} D_{1}}$ are also the angle-bisectors of $\widehat{a_{*} c_{*}}$. 


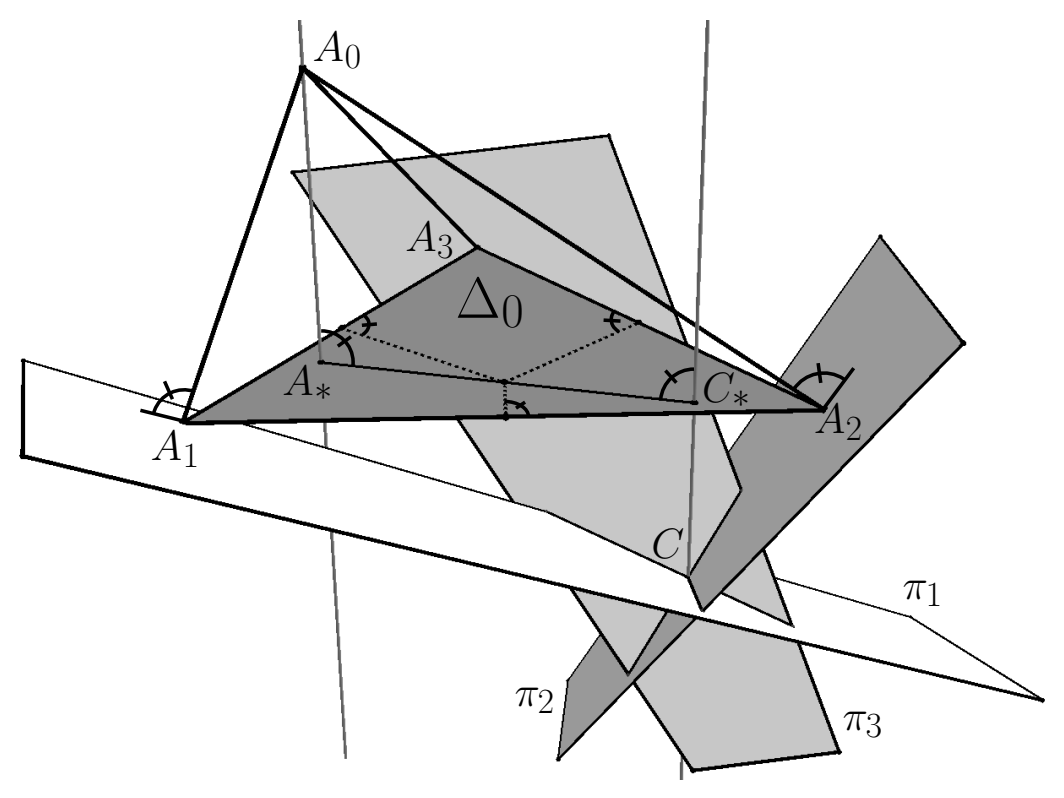

FIgURE 10. Three-dimensional version of Theorem 5.1

The previous construction is valid also for the elliptic case, and it can be checked that this theorem also holds in the Euclidean plane.

\section{HighER-DimENSIONAL GENERALIZATIONS}

It should be interesting to generalize the above techniques to higher dimensions: to find higher-dimensional generalizations of Theorems $1.2,4.2,4.3,4.4,4.5$ or 4.6 , perhaps in terms of generalized tetrahedra [12, 13].

A higher-dimensional generalization of the Euclidean version of Theorem 1.2 is:

Theorem 5.1. Let $\Delta$ be an n-simplex in Euclidean $n$-dimensional space with vertices $A_{0}, A_{1}, \ldots, A_{n}$. Consider the opposite face $\Delta_{0}$ to $A_{0}$ in $\Delta$, and take the hyperplane $\alpha_{0}$ containing $\Delta_{0}$. Let $\pi_{1}, \pi_{2}, \ldots, \pi_{n}$ be the hyperplanes orthogonal to $A_{0} A_{1}, A_{0} A_{2}, \ldots, A_{0} A_{n}$ through $A_{1}, A_{2}, \ldots, A_{n}$ respectively, and let $C$ be the intersection point of $\pi_{1}, \pi_{2}, \ldots, \pi_{n}$. If $A_{*}$ and $C_{*}$ are the orthogonal projections of $A_{0}$ and $C$ respectively into $\alpha_{0}$, then the midpoint of $\overline{A_{*} C_{*}}$ is the circumcenter of $\Delta_{0}$.

Proof. We proceed by induction on $n$.

For $n=2$, the result is given by the Euclidean version of Theorem 1.2 . We omit the proof.

Assume that the result is true for simplices in Euclidean $(n-1)$-dimensional space.

Let $\Delta$ be the simplex in Euclidean $n$-dimensional space of the statement. Let $M$ be the midpoint of $\overline{A_{*} C_{*}}$. For $i=1,2, \ldots, n$ let $\Delta_{i}^{\prime}$ be the opposite face of $A_{i}$ in 
$\Delta_{0}$, and let us denote $\pi_{i}^{\prime}=\pi_{i} \cap \alpha_{0}$. Since $\pi_{i}$ is orthogonal to $A_{0} A_{i}$, the line $A_{*} A_{i}$ is orthogonal to $\pi_{i}^{\prime}$ for $i=1,2, \ldots, n$.

Let $\alpha_{1}$ be the hyperplane containing $A_{0}, A_{2}, \ldots, A_{n}$, and consider $\alpha_{1}^{\prime}=\alpha_{0} \cap$ $\alpha_{1}$. Since $\pi_{1}, \pi_{2}, \ldots, \pi_{n}$ intersect in a point in Euclidean $n$-dimensional space, the intersection of the $(n-2)$-dimensional subspaces $\pi_{2}^{\prime} \cap \ldots \cap \pi_{n}^{\prime}$ is a point $C_{1}$ of the $(n-1)$-dimensional space $\alpha_{0}$. The line $C C_{1}$ coincides with $\pi_{2} \cap \ldots \cap \pi_{n}$. Hence, it is orthogonal to $\alpha_{1}$. This implies that $C_{*} C_{1}$, the orthogonal projection of $C C_{1}$ into $\alpha_{0}$, is orthogonal to $\alpha_{1}^{\prime}$.

Let $A_{*}^{1}$ and $C_{*}^{1}$ be the orthogonal projections of $A_{*}$ and $C_{*}$, respectively, into $\alpha_{1}^{\prime}$. Note that $C_{*}^{1}$ is also the orthogonal projection of $C_{1}$ into $\alpha_{1}^{\prime}$. Applying the induction hypothesis to the simplex in $\alpha_{0}$ with vertices $A_{*}, A_{2}, \ldots, A_{n}$, the midpoint of $\overline{A_{*}^{1} C_{*}^{1}}$ is the circumcenter of $\Delta_{1}^{\prime}$. Therefore, the orthogonal projection of $M$ into $\alpha_{1}^{\prime}$ is the circumcenter of $\Delta_{1}^{\prime}$, and this implies that $M$ is equidistant from $A_{2}, \ldots, A_{n}$. Proceeding in the same way, we conclude that $M$ is equidistant from $A_{1}, A_{2}, \ldots, A_{n}$, and $M$ is the circumcenter of $\Delta_{0}$.

The three-dimensional version of this theorem is illustrated in Fig. 10 We have checked that Theorem 5.1 is not valid in the 3-dimensional hyperbolic and elliptic cases. However, there are geometric constructions that are equivalent in Euclidean geometry but non-equivalent in the non-Euclidean case, and this implies that there are Euclidean concepts that have multiple non-Euclidean interpretations. For instance, it is well-known that the Euler line of a triangle, as it is usually defined in the Euclidean plane, does not exist in general for non-Euclidean triangles. Nevertheless, alternative definitions of the circumcenter and the barycenter of a triangle, different to the standard ones but equivalent to them in Euclidean geometry, can be given in such a way that the Euler line does exist for any triangle in the hyperbolic and elliptic planes [1, 15].

Question 5.2. Is there a different formulation of Theorem 5.1 which is valid also in the non-Euclidean cases?

It must be noted that in Euclidean $n$-space the set of points $A_{0}, A_{1}, \ldots, A_{n}, C$ of the statement of Theorem 5.1 is diametrically cyclic, in the sense that all these points lie in an $(n-1)$-dimensional sphere in $\mathbb{R}^{n}$ in which $A_{0}$ and $C$ are antipodal points, while this is not the case in the non-Euclidean context.

On the other hand, we have also tried to find an $n$-dimensional generalization of Theorem 4.6 without success.

Question 5.3. Is there an $n$-dimensional (Euclidean or non-Euclidean) version of Theorem 4.6?

\section{ACKNOWLEDGEMENTS}

The author wants to express his gratitude to Professors M. Avendano and A.M. Oller-Marcén for their valuable comments and suggestions during the writing of this paper. 


\section{REFERENCES}

[1] Akopyan, A. V., On some classical constructions extended to hyperbolic geometry, arXiv:1105.2153 [math.MG].

[2] Bachmann, F., Aufbau der Geometrie aus dem Spiegelungsbegriff. 2. Auflage, Springer, 1973. MR 0346643

[3] Bachmann, F., Ebene Spiegelungsgeometrie. Eine Vorlesung über Hjelmslev-Gruppen, Bibliographisches Institut, Mannheim, 1989. MR 1002943.

[4] Buser, P., Geometry and Spectra of Compact Riemann Surfaces, Birkhäuser, Boston, 1992. MR 1183224

[5] Coxeter, H. S. M., The Real Projective Plane, 2nd edition, Cambridge, 1955. MR 0070189

[6] Coxeter, H. S. M., Non-Euclidean Geometry, 6th edition, Mathematical Association of America, Washington, DC, 1998. MR 1628013

[7] Kline, M., Mathematical Thought from Ancient to Modern Times, Vol. 1, New Ed., Oxford University Press, 1990. MR 1039322

[8] Richter-Gebert, J., Perspectives on projective geometry. A guided tour through real and complex geometry, Springer, Heidelberg, 2011. MR 2791970

[9] Santaló, L., Geometrías No Euclidianas, Eudeba, Buenos Aires, 1961. MR 0177336

[10] Sharygin, I.F., Problems in Plane Geometry, MIR, Moscow, 1988.

[11] Thurston, W.P., The Geometry and Topology of 3-Manifolds, Lecture Notes, Dept. of Math., Princeton University Press, Princeton, NJ, 1979.

[12] Ushijima, A. The tilt formula for generalized simplices in hyperbolic space, Discrete Comput. Geom. 28 (2002), no. 1, 19-27. MR 1904007

[13] Ushijima, A. A volume formula for generalised hyperbolic tetrahedra, in Non-Euclidean Geometries, 249-265, Springer, New York, 2006. MR 2191251

[14] Veblen, O. and Young, J.W., Projective Geometry, vol. I, Boston, 1910; vol. II, Boston, 1918. MR 0179666

[15] Vigara, R., Non-Euclidean Shadows of Classical Projective Theorems, Springer, forthcoming.

[16] Zagier, D., A one-sentence proof that every prime $p \equiv 1(\bmod 4)$ is a sum of two squares, Amer. Math. Monthly 97 (1990), no. 2, 144.

\section{Rubén Vigara}

Centro Universitario de la Defensa - Zaragoza, Ctra. Huesca s/n, 50090 Zaragoza, Spain and I.U.M.A. - Universidad de Zaragoza, Edificio de Matemáticas, planta 1, Pedro Cerbuna, 12, 50009 Zaragoza, Spain

rvigara@unizar.es

Received: June 29, 2016

Accepted: July 24, 2017 